\title{
A multi-layer Classification Technique for High Resolution Satellite Images Using Radiometric Calibration Modelling
}

Original
Article

\author{
Mahmoud A. Shaqwky ${ }^{1}$, Ahmed S. Elsharkawy ${ }^{2}$, Essam H. Hamza ${ }^{1,}$ Hassan E. $^{2}$ \\ El-Hifnawy ${ }^{2}$
}

\author{
${ }^{1}$ Department of Electrical Engineering, Military Technical College, Cairo, Egypt \\ ${ }^{2}$ Dept. of Civil Engineering, Military Technical College, Cairo, Egypt
}

\begin{abstract}
Key Words:
A multi-layer classification technique, assessment of classification, classification, maximum likelihood (ML), reflectance

Corresponding Author:

Mahmoud A. Shaqwky, Dept. of electrical engineering, Military

Technical College, abdellam30@yahoo.com

Abstract

Recent developments in satellite sensors tend to the availability of high spatial and spectral resolution images. The motivation of this research paper is to get maximum benefits of different bands in high resolution satellite images. In this research paper, a novel classification technique is introduced where the shared texture features properties problem is addressed. Atmospheric correction is applied on a high resolution World View 2 (WV2) image to produce reflectance value for all spectral bands. Reflectance image is produced by knowing the environmental parameters of the images at the capturing time, which can be extracted from auxiliary files associated with the input image. A multi-layer classification tree analysis is applied on a reflectance image to extract urban area features based on investigated thresholding values. The proposed technique is investigated through MATLAB environment. The results of the proposed technique are assessed versus classification results of Maximum Likelihood classification technique that is applied through ENVI software. The assessment of classification results is represented in confusion matrix format and determination of Kappa Coefficient. The investigated technique succeeded in classifying urban area features up to $90 \%$. The proposed technique is fast, automated and suitable for any image with same spectral bands as WV2 satellite image.
\end{abstract}

\section{INTRODUCTION}

Satellite images play an important role in providing geographical information for many branches and applications as urban planning, agriculture and city development. Urban area features can be extracted from satellite image by performing supervised or unsupervised classification methods ${ }^{[1]}$. Maximum Likelihood Classifier (MLC) is one of the greatest techniques for mapping land use and gives accurate results ${ }^{[2,}$ 3]. High resolution World View 2 (WV2) satellite sensor provides images with multispectral data. The spectral bands are used in separating land use features by detecting spectral signatures of specific feature ${ }^{[4]}$. The electromagnetic radiation signals collected by satellites in the solar spectrum are modified by scattering and absorption by gases and aerosols while traveling through the atmosphere from the Earth surface to sensors. The effects of atmospheric correction are to reduce the error in estimating reflectance to set of multitemporal data which is very important factors in application of classification and change detection for remotely sensed data ${ }^{[5,6]}$. Using a calibrated high radiometric resolution sensor such as WV2 allows greater classification automation with reduced manual editing. WV2 satellite remote sensing data compared with aerial photographs for the purpose of discrimination features using object-based image analysis method and support vector machine algorithm give good results ${ }^{[7]}$. Multi-layer classification algorithm with fixed thresholds against multi-temporal high resolution satellite imagery is one of the most important techniques for monitoring place at different times. The training data from the same time and place are applied to classify remotely sensed data in another place and time. It is needed to obtain available information of sensor and study for spectral profile of classes in different bands well to provide automated and robust algorithm ${ }^{[8]}$. Radiometric calibration begins with a conversion to radiance, then to reflectance and classification results of the proposed tree analysis technique were compared using two band ratios that were evaluated by using four bands and Maximum Likelihood Classification Technique (MLC) for mapping urban area to five classes.

\section{AREA OF STUDY}

\section{A. Input Image}

The available data are three WV2 satellite images were captured on January $19^{\text {th }}, 2010$ covering an area from city of Rio De Janeiro. The data were 
downloaded from digital globe open source website. The projection of this image is UTM, with datum of WGS84 zone $23 \mathrm{~N}$. The area of study is approximate $4 \mathrm{~km}^{2}$ and bounded by coordinates as upper left corner coordinates are (lat $22^{\circ} 54^{\backslash} 7.79^{\prime \prime} \mathrm{S}$, lon $43^{\circ}$ $10^{\backslash} 38.09^{\|} \mathrm{W}$ ) and lower right corner coordinates of the image are (lat $22^{\circ} \quad 55^{\backslash} 11^{\|} \mathrm{S}$, lon $43^{\circ} \quad 9^{\backslash}$ $28.2471^{\prime \prime} \mathrm{W}$ ) the scenes are captured with different environments as shown in Table 1and figures 1,2 and 3

Table 1: Metadata of input images

\begin{tabular}{lccc}
\hline \hline data & I & II & III \\
\hline T & $13: 09: 5$ & $13: 10: 43.76$ & $13: 11: 58.76$ \\
& 3.5 & $63.7^{\circ}$ & $64^{\circ}$ \\
$B$ & $63.5^{\circ}$ & $7.5^{\circ}$ & $26.7^{\circ}$ \\
$H$ & $29.8^{\circ}$ & $7.6^{\circ}$ & $26.7^{\circ}$ \\
$m$ & $29.8^{\circ}$ & $0.0^{\circ}$ & $-2.3^{\circ}$ \\
$M$ & $1.6^{\circ}$ & & \\
\hline \hline
\end{tabular}

$\mathrm{T}=$ time of capturing image, $\mathrm{B}=$ Mean sun elevation angle, $\mathrm{H}=$ Mean Of nadir, $\mathrm{m}=$ Mean track angle, $\mathrm{M}=$ Mean cross angle

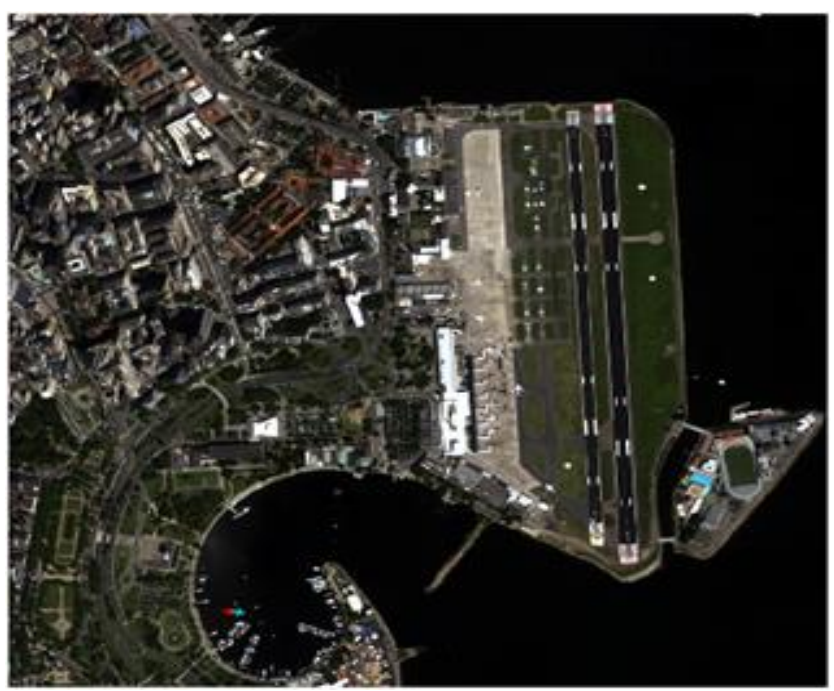

Fig. 1: Rio De Janeiro, WV-2 satellite image -dataset (I)

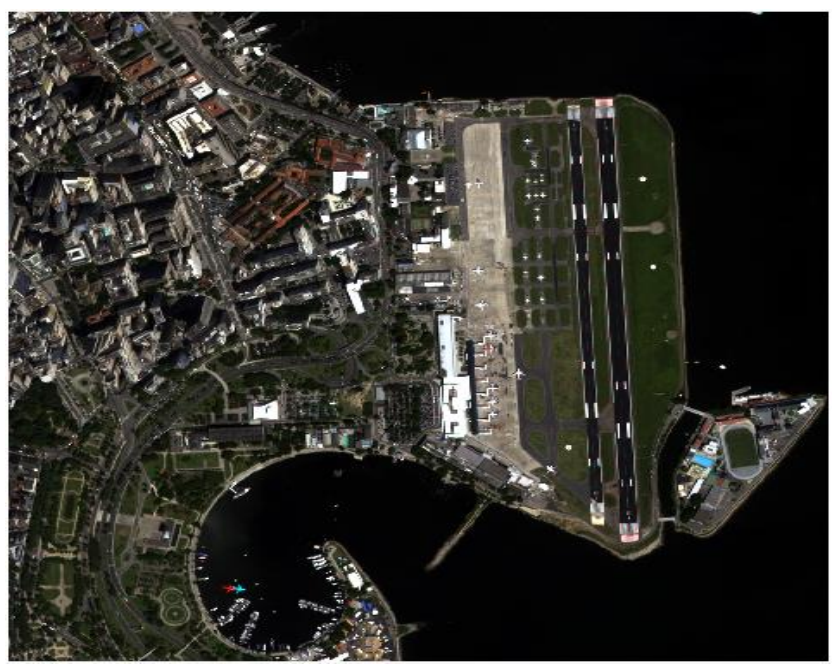

Fig. 2: Rio De Janeiro, WV-2 satellite image -dataset (II)

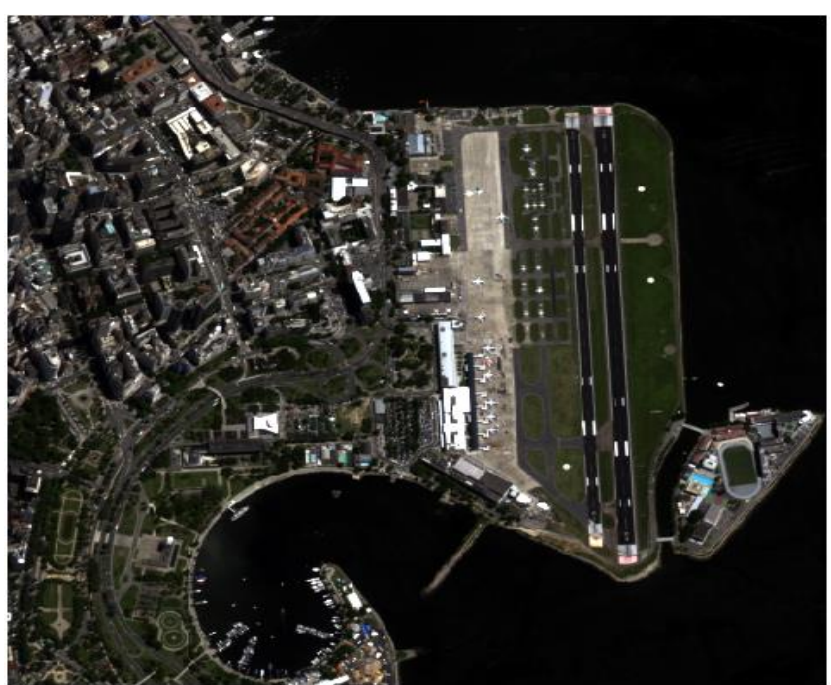

Fig. 3: Rio De Janeiro, WV-2 satellite image -dataset

B. WV2 Satellite Image:

In 2009, Digital Globe launched the WorldView-2 satellite which is the first commercial high-resolution satellite that provides eight spectral bands. Figure (4) is showing the advantages of WV2 satellite comparing with other satellites, Quick Bird, IKONOS and WorldView- $1^{[9]}$.

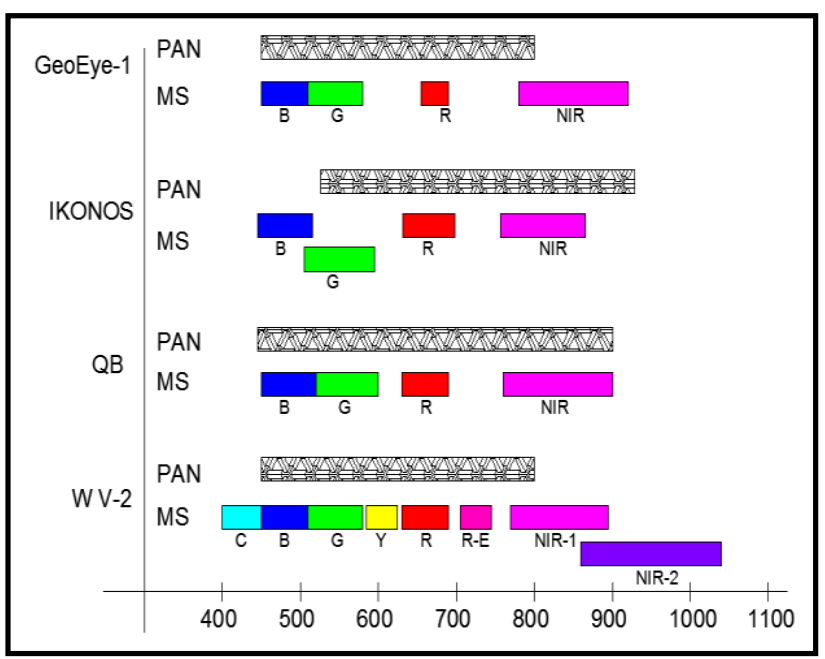

Fig. 3. Different spectrum band width OF WV2

\section{Multi Bands Specifications:}

WorldView-2 satellite provides four new unique spectral bands that give additional information for different features, Coastal blue, Yellow, Red edge and NIR-2 ${ }^{[10]}$. Coastal blue band supports vegetation identification and analysis and water penetration characteristics, and it is used to investigate radiometric calibration techniques. Yellow band is used to identify" yellow-ness" related to characteristics of targets, and it is important for vegetation applications. Also, yellow band assists in developing"true-color" hue correction for human vision representation. Red edge band is used to analyze vegetative 
condition directly related to plant health revealed through chlorophyll production. NIR-2 band overlaps the NIR-1 band but is less affected by atmospheric influence and it supports vegetation analysis and biomass studies ${ }^{[11]}$.

\section{RESEARCH METHODOLOGY}

This research work concentrated on classifying temporal data set of spectral bands from WV2 SATELLITES IMAGE USING A multi-layer classification Technique algorithm. Figure (5) describes the procedures that were applied in the experiment.

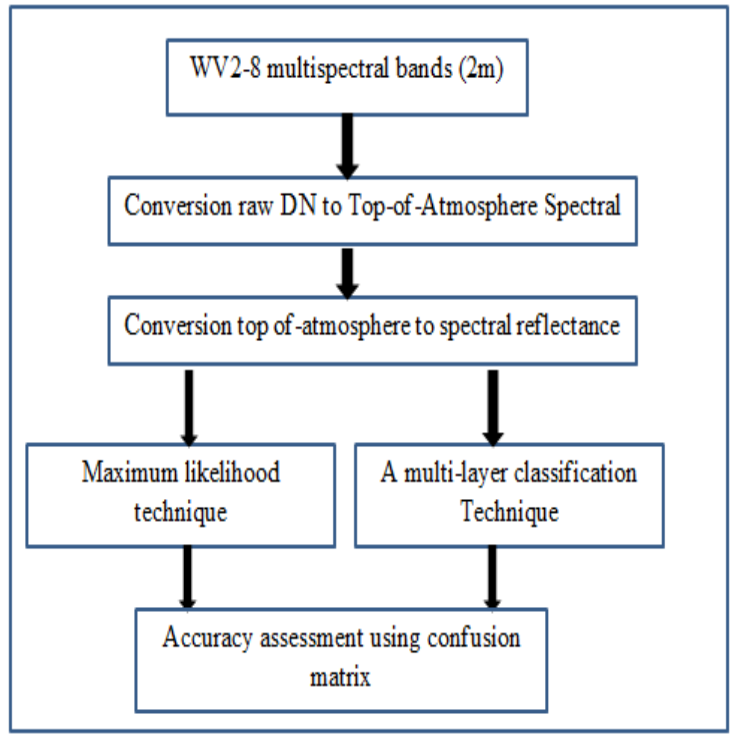

Fig. 5: Research methodology

Research begins with converting raw imagery (DNs) to atmosphere reflectance in order to account for the variation in relative positions between the sun, the earth and the satellite and to obtain an absolute value for the NDVI ratios or any indices which can be applied to any other scene [13]. The process of Converting the Digital Numbers (DN) to top of atmosphere (ToA) reflectance includes two main stages: First the DNs are converted to ToA radiance values. Then, these radiance values are converted to reflectance values.

\section{A. Conversion to Top-of-Atmosphere Spectral Radiance:}

WorldView-2 imagery is delivered to the customer as radiometrically corrected image pixels. The values of these pixels are calculated as a function of the amount of the spectral radiance enters the telescope aperture and the equipment conversion of that radiation into a digital signal ${ }^{[12]}$. Therefore, image pixel data are unique to WorldView-2 and should not be directly compared to imagery from other sensors in a radiometric/spectral sense. Top-of-atmosphere spectral radiance is defined as the spectral radiance entering the telescope aperture at the WorldView-2 altitude of $770 \mathrm{~km}$. The conversion from radiometrically corrected image pixels to spectral radiance according to Eq. (1) for each band of a WorldView-2 product $^{[13]}$ :

$$
\boldsymbol{L}_{\lambda_{\text {pixel,band }}}=\frac{\boldsymbol{k}_{\text {band }} \cdot \boldsymbol{q}_{\text {pixel,band }}}{\Delta \lambda_{\text {band }}}
$$

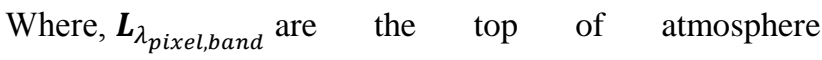
spectral radiance image pixels [W.m-2.sr-1. $\mu \mathrm{m}-1]$, $\boldsymbol{K}_{\text {band }}$ is the absolute radiometric calibration factor $\left[\mathrm{W} \cdot \mathrm{m}^{-2} \cdot \mathrm{sr}^{-1} \cdot\right.$ count $\left.^{-1}\right]$ for a given band; $\boldsymbol{q}_{\text {pixel,band }}$ are the given radiometrically corrected image pixels [counts]; $\boldsymbol{\Delta} \boldsymbol{\lambda}$ band is the effective bandwidth $[\mu \mathrm{m}]$ for a given band. Both $\boldsymbol{K}_{\text {band }}$ and $\boldsymbol{\Delta} \boldsymbol{\lambda}_{\text {band }}$ can be found in the image Metadata files (*.IDM) attached with the WorldView-2 product for each scenes under the names absCal Factor and effective bandwidth.

\section{B. Conversion to Top Of-Atmosphere Spectral Reflectance:}

After obtaining the ToA spectral radiance, this top of atmosphere spectral radiance varies with solar zenith angle, Earth-Sun distance, topography, bidirectional reflectance distribution function (BRDFthe target reflectance varies depending on the illumination and observation geometry), and atmospheric effects (absorption and scattering) ${ }^{[13]}$. So, that multispectral data must be converted into reflectance prior to performing spectral analysis techniques such as band ratios, Normalized Difference Vegetation Index (NDVI). There are several factors that must be known for each scene: the distance between the sun and earth in astronomical units, the day of the year (Julian date), and solar zenith angle.

$\mathrm{JD}=\operatorname{int}[356.25 .(\mathrm{year}+4716)]+\mathrm{int}[30.6001 .(\operatorname{month}+1)]+\mathrm{day}+\frac{\mathrm{ut}}{24}+\mathrm{B}-$ 1524.5

$\mathrm{dES}=1.00014-0.01671 \cdot \cos (\mathrm{g})-0.00014 \cdot \cos (2 \mathrm{~g})$

The Earth-Sun distance is calculated according to Eq. 3 will be in Astronomical Units (AU) and should have a value between 0.983 and 1.017. For the WorldView-2 launch date, October 8, 2009 at 18:51:00 GMT corresponds to the Julian Day 2455113.285; the Earth-Sun distance is 0.998987 AU. At least, six decimal places should be carried in the Earth-Sun distance for use in radiometric balancing or top-of atmosphere reflectance calculations $s^{[13]}$. The average solar Zenith angle has to be calculated for the whole scene at the time of acquisition according table(1) uses the following equation (4)

$$
\theta s=90.0-\operatorname{sun} E L
$$

Where the sunEl value can be found in file *.IDM. Finally, the process of converting the radiance 
values to reflectance values is performed according to the following equation:

$\rho_{\lambda_{\text {pixel,band }}}=\frac{L_{\text {pixel,band } .} d_{E S}^{2} \cdot \pi}{E_{S U N} \lambda_{\text {band }} \cdot \cos (\theta s)}$

Where $\rho_{\lambda_{\text {pixel,band }}}$ are the ToA reflectance values; $\mathrm{L}_{\text {pixel,band. are the ToA radiance values } \mathrm{dES}}$ is the Earth-Sun distance in Astronomical Units (AU) $\quad \mathrm{E}_{\mathrm{SUN}} \lambda_{\text {band }}$ WorldView-2 Band-Averaged Solar Spectral Irradiance $\theta_{s}$ The average solar Zenith angle.

The study uses two different methods with three data sets (I, II, III) for classifying land cover information:

1- Supervised classification approach using the Maximum Likelihood Classifier.

2- Image classification using multi-layer classification tree analysis. In the following sections, the details of these methods will be demonstrated.

C. Supervised Classification Approach using the Maximum Likelihood Classifier

For the purpose of applying Maximum Likelihood Classifier training pixels for five classes; asphalt roads, vegetation, building, shadow and water were chosen for the three data sets (I, II, III) to define spectral signatures to each class through region of interest (ROI) where the best classification result was obtained with higher samples per class [14]. Maximum Likelihood decision rule is based on the probability that a pixel belongs to a particular class. The basic equation assumes that these probabilities are equal for all classes. Maximum Likelihood Classifier is performed on the 8multispectral reflectance input bands of three datasets (I,II,III) uses the following Eq. $6^{[15]}$

$\begin{aligned} & \mathrm{D} \\ & \mathrm{Mc})]\end{aligned}=\ln (\mathrm{ac})-[0.5 \ln (|\operatorname{Covc}|)]-[0.5(\mathrm{X}-\mathrm{Mc}) \mathrm{T}(\operatorname{Cov}-1)(\mathrm{X}-$

Where: $\mathrm{D}=$ weighted distance (likelihood), $\mathrm{c}=\mathrm{a}$ particular class, $X=$ measurement vector of the candidate pixel, $\mathrm{Mc}=$ mean vector of the sample of class $\mathrm{c}, \mathrm{ac}=$ percent probability that any candidate pixel is a member of class c,(Defaults to 1.0, or is entered from a priori knowledge), Covc = covariance matrix of the pixels in the sample of class c, $|\mathrm{Covc}|=$ determinant of Covariance (matrix algebra),Covc-1 = inverse of Covariance (matrix algebra) $\ln =$ natural logarithm function.

\section{Supervised Classification Approach using the New Band Ratios}

Generally, The NDVI ratio in addition to one new band ratios are introduced, the original NDVI (R1) especially suited for vegetation and water, second one (R2) to detect asphalt roads, shadow and building. This equation developed by taking random points in study area for classes and uses the Spectral Profile to visualize the reflectance spectrum of a single pixel through many bands using these points. A careful study of spectral profile allowed to estimate the chemical composition of the material in the spectral bands and select yellow and green bands which contain high spectral information about classes. Table 2 summarizes the two ratios and their usage.

\begin{tabular}{|c|c|c|}
\hline$\overline{\text { indices }}$ & Band ratio & Target classes \\
\hline $\mathbf{R 1}$ & $\frac{\mathrm{R}-\mathrm{NIR} 1}{\mathrm{R}+\mathrm{NIR} 1}$ & $\begin{array}{l}\text { Vegetation } \\
\text { Water }\end{array}$ \\
\hline $\mathbf{R 2}$ & $\frac{\mathrm{CB}-\mathrm{NIR} 2}{\mathrm{CB}-\mathrm{NIR} 2}$ & $\begin{array}{l}\text { Shadow } \\
\text { Roads } \\
\text { Building }\end{array}$ \\
\hline
\end{tabular}

R1 is applied first to separate the image into vegetation and non-vegetation within lower and upper thresholds, and water is detected above certain threshold and then $\mathrm{R} 2$ ratio is applied to detect shadow above certain threshold, finally the roads and buildings are detected above certain threshold. Figure (6) summarize these steps along with the applied thresholds.

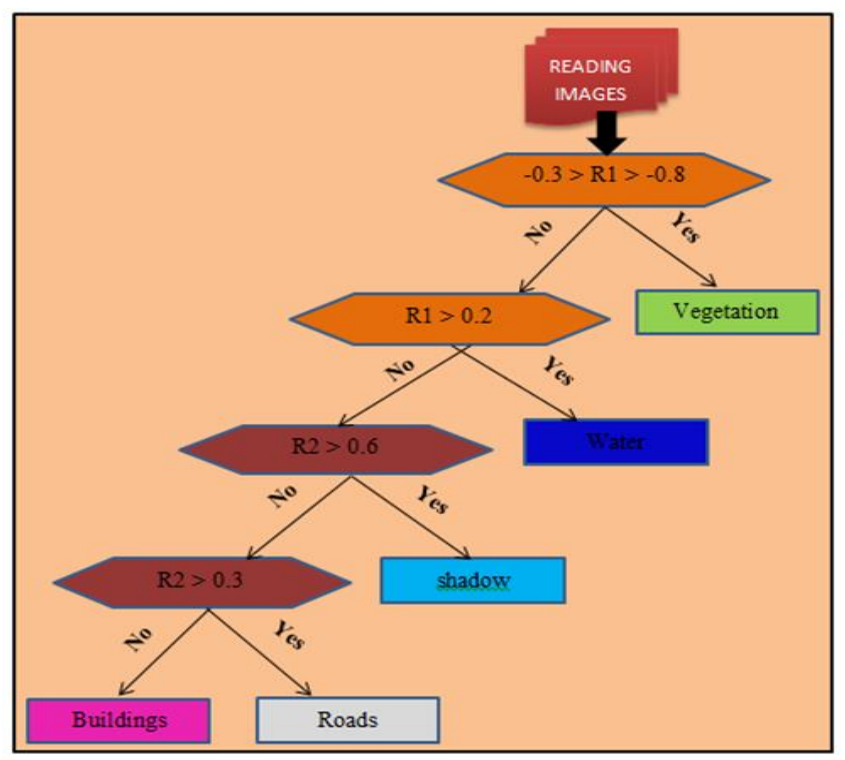

Fig. 4: Applying the band ratios with the proposed thresholds

\section{DISCUSSION AND ANALYSIS RESULTS}

Accuracy assessment of the MLC classification and Decision Tree classifier were calculated by means of confusion matrix (sometimes called error matrix), which compute the relationship between results of a classification and the corresponding reference data (ground truth) .We obtain from accuracy assessment report that contains producer and user accuracies, an overall accuracy, kappa coefficient $^{[16]}$. The user's accuracy refers to the measure of commission errors that correspond to those pixels from other classes that the classifier has labelled as belonging to the class of interest. Moreover, the producer's accuracy refers to the 
measure of omission errors that correspond to those pixels belonging to a class of interest that the classifier didn't recognize. kappa coefficient is another method for measuring accuracy of classification which is calculated by subtracting Chance agreement incorporates off-diagonal from Observed accuracy determined by diagonal in error matrix and divided by subtracting Chance agreement from one ${ }^{[17]}$. The classification results from the Decision Tree and MLC classifier were compared with ground truth pixels. The confusion matrices with the overall Accuracy and the Kappa Coefficient of the datasets I, II, III are displayed in Tables as follows:

Table 3: Confusion matrix of the datasets (I) using "MLC" with ROI ground truth pixels, Overall Accuracy $=91.4544 \%$, Kappa Coefficient $=0.8697$

\begin{tabular}{lccccccccc}
\hline \hline & VEG & W & SH & R & B & T & $\begin{array}{c}\text { P. } \\
\text { ACC }\end{array}$ & $\begin{array}{c}\text { U. } \\
\text { ACC }\end{array}$ \\
\hline \hline VEG & 97.10 & 0.01 & 0.47 & 1.35 & 0.55 & 18.47 & 97.10 & 98.52 \\
W & 0.00 & 99.52 & 0.00 & 0.00 & 0.00 & 52.79 & 99.52 & 100.00 \\
SH & 0.09 & 0.31 & 94.10 & 30.51 & 2.97 & 7.16 & 94.10 & 32.03 \\
R & 1.75 & 0.16 & 4.07 & 60.04 & 13.08 & 10.57 & 60.04 & 80.87 \\
B & 1.05 & 0.00 & 1.36 & 8.10 & 83.39 & 11.01 & 83.39 & 87.41 \\
T & 100.00 & 100.00 & 100.00 & 100.00 & 100.00 & 100.00 & & \\
\hline \hline
\end{tabular}

The SYMBOLS according tables as: $\mathrm{VEG}=$ vegetation, $\mathrm{W}=$ water, $\mathrm{SH}=$ shadow, $\mathrm{R}=$ roads, $\mathrm{B}=$ building, $\mathrm{T}=$ sum, $\mathrm{P} . \mathrm{ACC}=$ producer accuracy and U.ACC=user accuracy.

Table 4: Confusion matrix of the datasets (I) using "tree analysis" with ROI ground truth, Overall Accuracy $=90.9435 \%$, Kappa Coefficient $=0.8632$

\begin{tabular}{|c|c|c|c|c|c|c|c|c|}
\hline & "VEG & $\overline{\mathrm{WW}}$ & 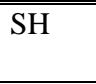 & 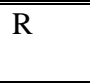 & $\overline{\mathrm{B}}$ & $\overline{\overline{T T}}$ & $\begin{array}{c}\text { P. } \\
\text { ACC }\end{array}$ & $\begin{array}{c}\mathrm{U} . \\
\mathrm{ACC}\end{array}$ \\
\hline 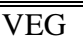 & 96.10 & "0.01 & "0.06 & 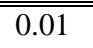 & $\overline{c 0.34}$ & 18.19 & 96.10 & $\begin{array}{l}99.01 \\
\end{array}$ \\
\hline W & 0.00 & 95.89 & 0.06 & 0.01 & 0.04 & 50.87 & 95.89 & 99.99 \\
\hline $\mathrm{SH}$ & 0.02 & 4.08 & 84.72 & 11.58 & 1.94 & 6.11 & 84.72 & 33.80 \\
\hline $\mathrm{R}$ & 1.32 & 0.01 & 14.60 & 76.74 & 19.03 & 13.73 & 76.74 & 79.56 \\
\hline B & 2.56 & 0.00 & 0.19 & 10.79 & 78.65 & 11.09 & 78.65 & 81.78 \\
\hline $\mathrm{T}$ & 100 & 100 & 100. & 100 & 100 & 100 & & \\
\hline
\end{tabular}

Table 5: Confusion matrix of the data set (II) using "MLC" with ROI ground truth pixels, Overall Accuracy = 91.4034\%, Kappa Coefficient $=0.8539$

\begin{tabular}{lcccccccc}
\hline \hline & VEG & W & SH & R & B & T & $\begin{array}{c}\text { P. } \\
\text { ACC }\end{array}$ & $\begin{array}{l}\text { U ACC } \\
\text { ACG }\end{array}$ \\
& 97.89 & 0.01 & 0.13 & 0.95 & 0.36 & 17.53 & 97.89 & 99.16 \\
W & 0.00 & 96.55 & 0.06 & 0.00 & 0.00 & 59.31 & 96.55 & 100.00 \\
SH & 0.06 & 2.24 & 93.98 & 41.50 & 0.57 & 7.45 & 93.98 & 16.48 \\
R & 1.31 & 1.15 & 5.04 & 52.16 & 5.39 & 7.46 & 52.16 & 80.72 \\
B & 0.74 & 0.05 & 0.79 & 5.39 & 93.68 & 8.26 & 93.68 & 90.40 \\
$\mathrm{~T}$ & 100 & 100 & 100. & 100 & 100 & 100 & & \\
\hline \hline
\end{tabular}


Table 6: Confusion matrix of the data set (II) using "tree analysis" with ROI, Overall Accuracy $=93.3816 \%$, Kappa Coefficient $=$ 0.8888

\begin{tabular}{lcccccccc}
\hline \hline & VEG & W & SH & R & B & T & $\begin{array}{c}\text { P. } \\
\text { ACC }\end{array}$ & $\begin{array}{c}\text { U. } \\
\text { ACC }\end{array}$ \\
\hline \hline VEG & 96.51 & 0.00 & 0.13 & 0.53 & 0.14 & 17.22 & 96.51 \\
W & 0.00 & 93.53 & 0.22 & 0.00 & 0.10 & 57.46 & 93.53 \\
SH & 0.00 & 6.41 & 80.63 & 0.02 & 0.18 & 5.01 & 80.63 & 21.02 \\
R & 1.01 & 0.04 & 18.45 & 91.94 & 10.11 & 11.86 & 91.94 & 89.43 \\
B & 2.47 & 0.02 & 0.57 & 0.02 & 89.47 & 8.46 & 89.47 \\
T & 100 & 100 & 100 & 100 & 100 & & 84.32 \\
\hline \hline
\end{tabular}

Table 7: Confusion matrix of the data set (III) using "MLC" with ROI ground truth, Overall Accuracy $=92.2678 \%$, Kappa Coefficient $=$ 0.8738

\begin{tabular}{|c|c|c|c|c|c|c|c|c|}
\hline & " VEG & "W & $\overline{\mathrm{SH}}$ & R & B & $\mathrm{T}$ & $\begin{array}{l}\mathrm{P} . \\
\mathrm{ACC}\end{array}$ & $\begin{array}{l}\mathrm{U} . \\
\text { ACC }\end{array}$ \\
\hline $\begin{array}{l}\mathrm{VE} \\
\mathrm{G}\end{array}$ & 97.29 & 0.01 & 0.60 & $\begin{array}{l}1.10 \\
\end{array}$ & 0.85 & 21.08 & 97.29 & 999.03 \\
\hline W & 0.00 & 98.34 & 0.00 & 0.00 & 0.00 & 56.51 & 98.34 & 100.00 \\
\hline SH & 0.21 & 0.66 & 96.14 & 36.48 & 0.58 & 5.56 & 96.14 & 24.96 \\
\hline $\mathrm{R}$ & 0.84 & 0.97 & 3.13 & 55.77 & 16.06 & 7.96 & 55.77 & 71.02 \\
\hline B & 1.66 & 0.03 & 0.13 & 6.65 & 82.51 & 8.88 & 82.51 & 88.21 \\
\hline $\mathrm{T}$ & 100 & 100 & 100 & 100 & 100 & 100 & & \\
\hline
\end{tabular}

Table 8: Confusion matrix of the data set (III) using "tree analysis" with ROI ground, Overall Accuracy $=90.3131 \%$, Kappa Coefficient $=0.8476$

\begin{tabular}{lllllllcr}
\hline \hline & VEG & W & SH & R & B & T & $\begin{array}{c}\text { P. } \\
\text { ACC }\end{array}$ & $\begin{array}{c}\text { U. } \\
\text { ACC }\end{array}$ \\
\hline \hline VE & 96.01 & 0.00 & 0.55 & 0.61 & 0.57 & 20.73 & 96.01 \\
G & 0.00 & 90.55 & 0.00 & 0.00 & 0.06 & 52.04 & 90.55 & 99.99 \\
W & 0.01 & 9.42 & 82.94 & 0.26 & 0.26 & 6.66 & 82.94 & 17.98 \\
SH & 0.82 & 0.03 & 16.23 & 73.93 & 4.52 & 8.35 & 73.93 & 89.74 \\
R & 3.16 & 0.00 & 0.29 & 73.93 & 94.59 & 12.22 & 94.59 \\
B & 100 & 100 & 100 & 100 & 100 & 100 & 73.52 \\
T & & & & & & & \\
\hline \hline
\end{tabular}


Figures (6), (7), (8), (9), (10), (11) and (12), illustrate the overall accuracy and classification results for the data sets using two classifier comparisons, respectively.

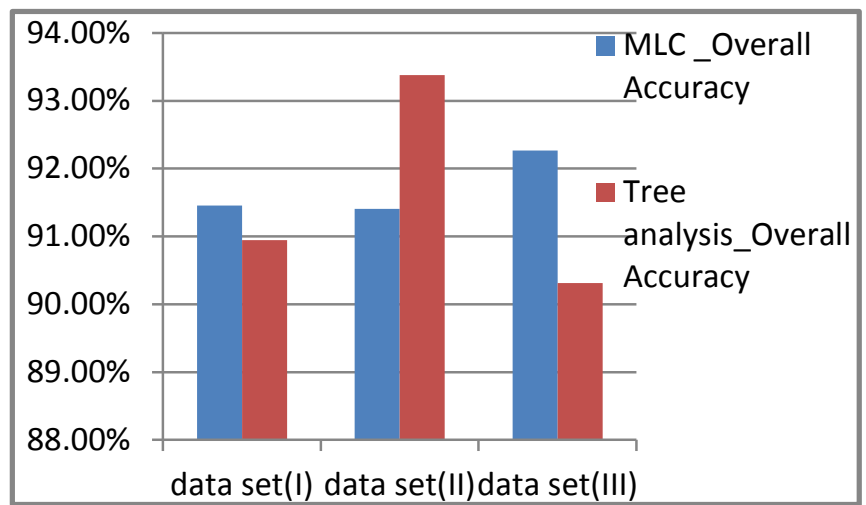

Fig 7: Over all accuracy classification results for datasets

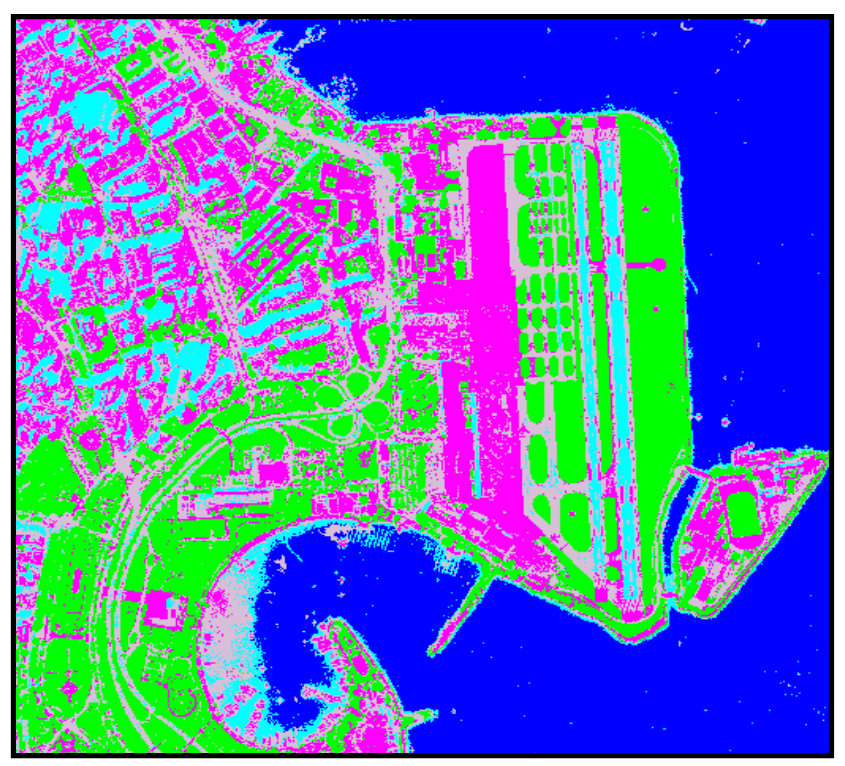

Fig. 8: MLC results for Data (I)

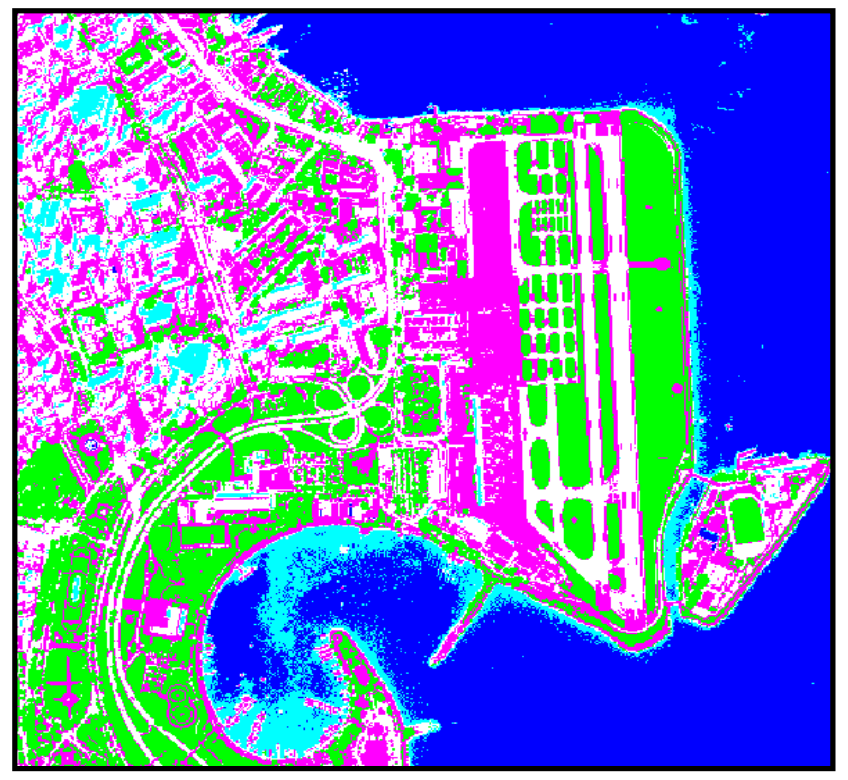

Fig. 9: Tree analysis results for Data (I)

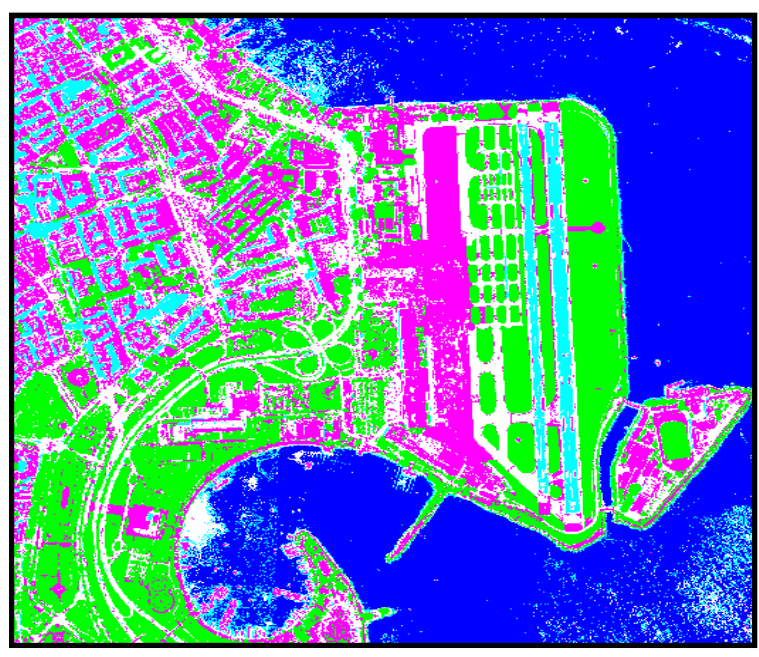

Fig. 10: MLC results for Data (II)
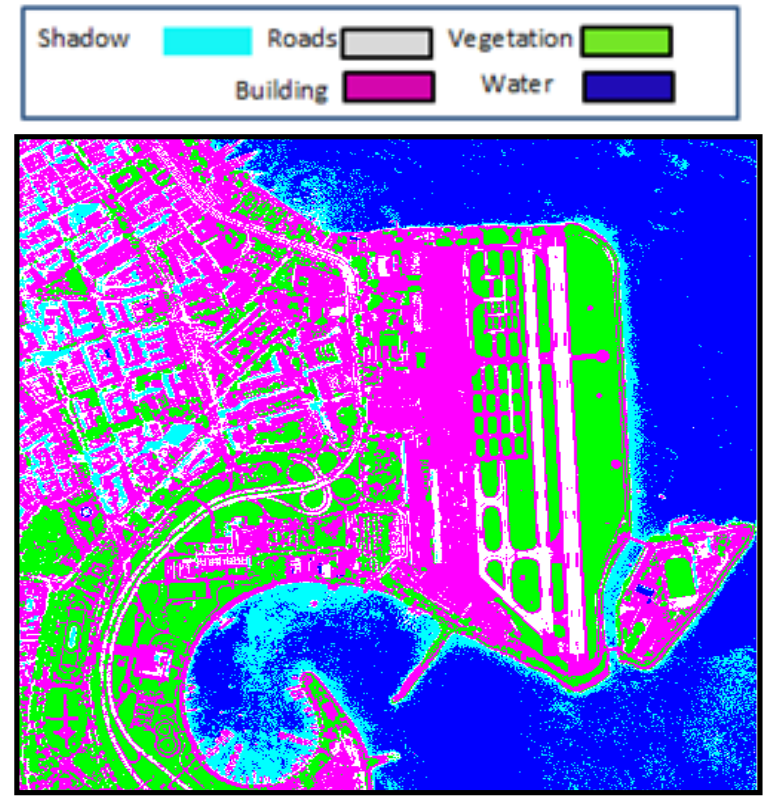

Fig. 11: Tree analysis results for Data (II)

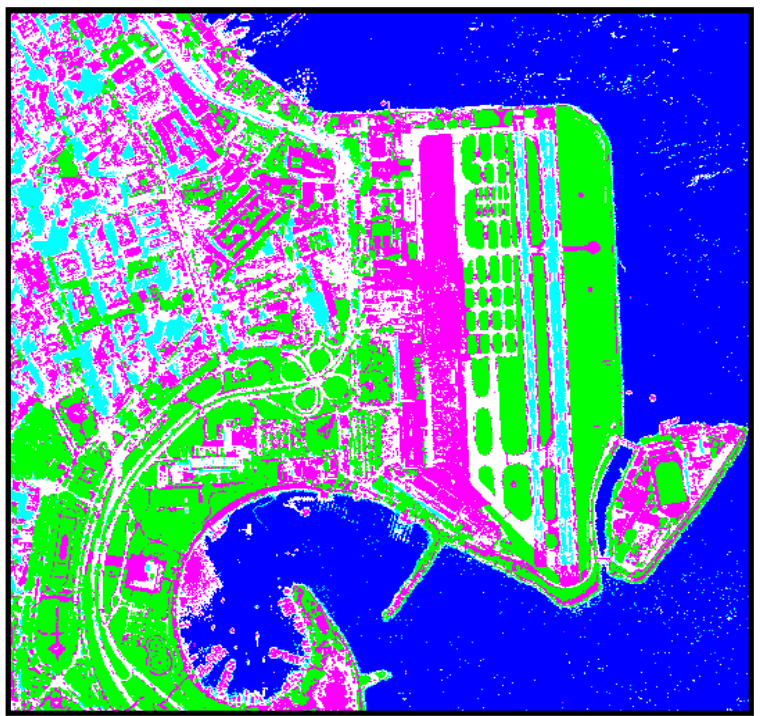

Fig .12: MLC results for Data (III)

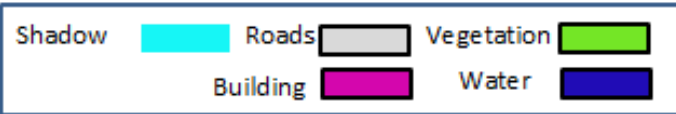




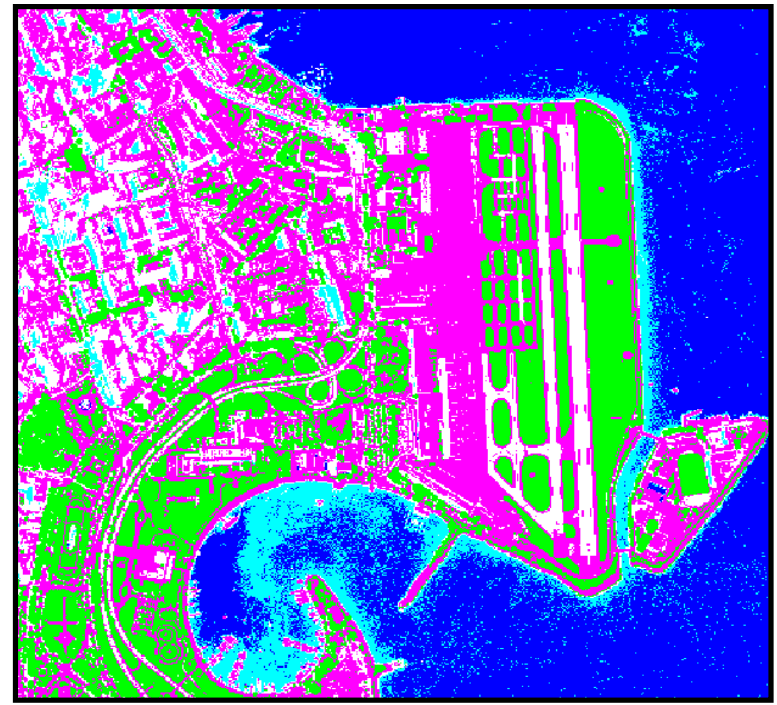

Fig. 13: Tree analysis results for Data (III)

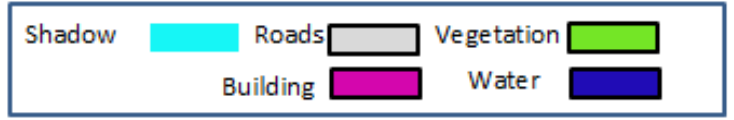

A careful study of these results showed that three datasets yielded excellent producer and user accuracies for all classes when using the same classification thresholds after applying the radiometric normalization. Multi-layer classification approach is higher than MLC in classifying manmade objects and separating between two classes shadow and asphalt roads.

\section{CONCLUSION}

In this study, the relative radiometric calibration process for multi-temporal WorldView-2 data was evaluated by applying a multi-layer classification algorithm with fixed thresholds (versus) MLC for three datasets of multi-temporal high resolution satellite images for study area over Rio de Janeiro. The overall accuracies of classification results against three datasets of multi-temporal high resolution satellite imagery using two classification techniques are above $90 \%$ and kappa coefficients above 0.8. The results of this investigation show very good potentials and automated approach for the use of a multi-layer classification algorithm with fixed thresholds after applying the relative radiometric normalization to mapping land use and give better result than MLC for separating interference problems between manmade objects or classes such as building, shadow and roads also extract it.

\section{REFERENCES}

[1] Abburu, S. and S.B. Golla," Satellite image classification methods and techniques: A review." International journal of computer applications", 2015. 119(8).

[2]Madhura $M$ and S. Venkatachalam, "Comparison of Supervised Classification Methods On Remote Sensed Satellite Data: An Application In Chennai, South India”. International Journal of Science and Research (IJSR), February 2015. 4(2): p. 5.

[3]Mahmoud A. Shawky, et al., 'Investigation of Optimum Spectral Bands forUrban Area Classification from World View2 (WV2) Satellite Image". International Journal of Innovative Research in Computerand Communication Engineering, 2016. 4(9): p. 15701- 15707.

[4]Tarantino, C., et al.," 8-band image data processing of the worldview-2 satellite in a wide area of applications". 2012: INTECH Open Access Publisher.

[5]Song, C., et al., "Classification and change detection using Landsat TM data: when and how to correct atmospheric effects?Remote sensing of Environment", 2001. 75(2): p. 230-244.

[6]El Hajj, M., et al., "Relative radiometric normalization and atmospheric correction of a SPOT 5 time series". Sensors, 2008. 8(4): p. 2774-2791.

[7]Heenkenda, M.K., et al., Mangrove species identification: 'Comparing WorldView-2 with aerial photographs. Remote Sensing", 2014. 6(7): p. 6064-6088.

[8] Elhabiby, M., A. Elsharkawy, and N. El-Sheimy, "Assessment Of Relative Atmospheric Normalization For MultiTemporal

Assessment', 2012. 1(2): p. 01-10.

[9]Elsharkawy, A. "A modified parallelepiped-like method for supervised classification for high resolution satellite imagery". in CGU Annual Scientific Meeting, Banff, Calgary. 2011.

[10] Elsharkawy, A., M. Elhabiby, and N. El-Sheimy," New Combined Pixel/Object-Based Technique for Efficient Urban Classification Using Worldview-2 Data'. International Archives of the Photogrammetry, Remote Sensing and Spatial Information Sciences, 2012. 39: p. B7.

[11] Data sheets from http://www.digitalglobe.com.

[12] Globe, D., DigitalGlobe Core Imagery Products Guide. ed, 2009.

[13]Updike, T. and C. Comp, "Radiometric use of WorldView-2 imagery'. Technical Note, 2010: p. 1-17.

[14]Rego, L.F.G. and B. Koch." Automatic classification of land cover with high resolution data of the Rio de Janeiro City Brazil. in Remote Sensing and Data Fusion over Urban Areas", 2003. 2nd GRSS/ISPRS Joint Workshop on. 2003. IEEE.

[15]Asmala, A.," Analysis of maximum likelihood classification on multispectral data". Applied Mathematical Sciences, 2012. 6(129-132): p. 6425-6436.

[16] Congalton, R.G., "A review of assessing the accuracy of classifications of remotely sensed data. Remote sensing of environment", 1991. 37(1): p. 35-46.

[17] Richards, J.A. and J. Richards, "Remote sensing digital image analysis. Vol. 3. 1999: Springer. 